\title{
Muscle and adipose tissue biopsy in older adults with type 2 diabetes
}

\author{
Yi Wang ${ }^{1}$, David Simar ${ }^{2}$, Kylie Anderberg ${ }^{1}$, Yorgi Mavros ${ }^{1}$, Shelley Kay ${ }^{1}$, Renru Zhao ${ }^{1}$, \\ Bernhard T. Baune ${ }^{3}$, Anthony O'Sullivan ${ }^{4}$, Nalin Singh ${ }^{1,5,6}$, Maria Fiatarone Singh $^{1,7,8^{*}}$ \\ ${ }^{1}$ Exercise, Health and Performance Research Group, Faculty of Health Sciences, University of Sydney, Sydney, Australia; \\ ${ }^{2}$ School of Medical Sciences, University of New South Wales, Sydney, Australia; \\ ${ }^{3}$ The University of Adelaide, Adelaide, Australia; \\ ${ }^{4}$ Departments of Medicine and Endocrinology, St George Hospital, Sydney, Australia; \\ ${ }^{5}$ The Centre for STRONG Medicine, Balmain Hospital, Sydney, Australia; \\ ${ }^{6}$ Royal Prince Alfred Hospital, Sydney, Australia; \\ ${ }^{7}$ Sydney Medical School, University of Sydney, Sydney, Australia; ${ }^{*}$ Corresponding Author: maria.fiataronesingh@,sydney.edu.au \\ ${ }^{8}$ Hebrew SeniorLife and Jean Mayer USDA Human Nutrition Centre on Aging, Tufts University, Boston, USA
}

Received 27 April 2011; revised 20 May 2011; accepted 29 May 2011.

\begin{abstract}
We describe a method for obtaining skeletal muscle and subcutaneous adipose tissue in a single percutaneous needle biopsy procedure. Biopsies were performed in a double-blind randomized, sham-exercise controlled clinical trial of power training in 103 older adults with type 2 diabetes. In total 110 biopsies (73.3\%) were performed in the first 50 participants at 3 time points. Muscle samples were obtained in 102/110 biopsies (92.7\%); adipose samples were obtained in 72/110 biopsies (65.5\%); both tissue types were obtained in 65/110 biopsies (59.1\%). The average weight of muscle samples was $131 \pm 92 \mathrm{mg}$ and the median weight of adipose samples was $51 \mathrm{mg}$ (range 3 to $412 \mathrm{mg}$ ). No adverse effects of biopsies were reported other than mild transient pain or light-headedness. This is one of the first reports of both adipose and muscle tissue obtained during the same biopsy, and the first to describe in detail sampling technique, yield and safety.
\end{abstract}

Keywords: Biopsy; Skeletal Muscle; Subcutaneous Adipose Tissue; Type 2 Diabetes

\section{INTRODUCTION}

Alterations in the amount, distribution, and metabolic profile and hormonal pathways of both skeletal muscle and adipose tissue in individuals with type 2 diabetes are increasingly recognized as central to the pathophysiology of this disorder [1]. For example, $90 \%$ of individuals with type 2 diabetes are obese [2], and excess adipocytes accumulation is primarily central (subcutaneous and visceral truncal) in distribution [3]. Excess visceral adiposity is one of the most important abnormalities linking insulin resistance, type 2 diabetes, metabolic syndrome, systemic inflammation, and the vastly elevated risk of cardiovascular morbidity and mortality in this cohort [4]. Recent evidence has revealed several biological and genetic differences between intra-abdominal visceral fat and peripheral (appendicular) subcutaneous fat. For example, visceral adipose tissue floods the portal circulation with free fatty acids (FFA), thus exposing non-adipose tissues to FFA excess. This is accompanied by increased triglyceride (TG) accumulation in muscles, liver and pancreatic beta-cells, resulting in both insulin resistance and beta-cell dysfunction/insulin deficiency [3]. Moreover, visceral adipose tissue (via macrophages residing in this fat depot) produces pro-inflammatory cytokines including tumor necrosis factor-alpha (TNF- $\alpha$ ) and interleukin-6 (IL-6) and contributes to less production of anti-inflammatory adiponectin than other adipose depots [5]. These cytokines imbalances contribute to insulin resistance and play a major role in the pathogenesis of endothelial dysfunction and subsequent atherosclerosis [6]. In contrast, peripheral subcutaneous adipose tissue performs a vital role of buffering fluxes of FFA in the circulation [3]. There is also evidence suggesting that the subcutaneous fat plays an important role in modulating peripheral insulin resistance by regulating visceral fat accumulation [6]. For example, in animals, peripheral lipectomy (reducing the number of subcutaneous adipocytes) causes increased lipid deposition in visceral adipose tissue, muscle and liver with metabolic consequences similar to the insulin resistance syndrome [3]. It has also been reported that bilateral removal of 
subcutaneous inguinal fat pads in mice resulted in increased accumulation of visceral fat and TNF- $\alpha$ expression, decreased insulin sensitivity and Tyrosine phosphorylation of IRS-1. These abnormalities were corrected after re-implantation of inguinal fat [7,8]. However, the role of subcutaneous adipose tissue in insulin resistance, type 2 diabetes, metabolic syndrome, systemic inflammation, and cardiovascular morbidity and mortality, and its adaptation to interventions such as diet and exercise in human are not fully understood. Liposuction in human has contradictory effects on insulin sensitivity. For example, Giugliano [9] and González [10] reported that subcutaneous liposuction in obese women led to an improvement in insulin sensitivity. On the other hand, Ybarra [11] and Robles-Cervantes [12] found that subcutaneous liposuction was not related to insulin sensitivity in non-diabetic overweight or obese women. However, liposuction in humans is usually the removal of both abdominal and peripheral subcutaneous adipose tissues, in contrast to the animal studies that specifically performed lipectomy in peripheral subcutaneous adipose tissues. On the other hand, weight loss by diet/exercise reduces both of these subcutaneous fat depots, and importantly selectively targets visceral fat as well [13]. Thus, studies of subcutaneous fat itself in relation to regional and whole body metabolism in those with type 2 diabetes and related syndromes are of great interest.

Although skeletal muscle biopsy has been used for many years in adults with type 2 diabetes to understand the factors related to insulin resistance such as muscle fiber distribution, glycogen storage, insulin signaling, oxidative and glycolytic enzyme capacity, markers of inflammation, and lipid metabolism [14], there is only one publication in English to our knowledge describing simultaneous muscle and subcutaneous adipose tissue biopsies in this or any cohort [15].

Therefore, the purpose of this investigation was to introduce a simple and novel method of obtaining both skeletal muscle and subcutaneous adipose tissue in one biopsy through the same incision, for the purpose of indentifying the roles of both muscle and peripheral subcutaneous fat in the pathogenesis and treatment of obesity, insulin resistance, and type 2 diabetes.

\section{METHODS}

\subsection{Study Design}

Biopsies were performed within a double-blind randomized, sham-exercise controlled clinical trial of power training in 103 older adults with type 2 diabetes. Ethical approval was obtained from Ethics Review Committee (Royal Prince Alfred Hospital (RPAH) Zone), Sydney South West Area Health Service (Ethics Committee
Protocol No: X04-0096) and written informed consent was obtained from all participants. The trial was registered with the Australian Clinical Trials Registry (ACTR) (ACTR No: ACTRN12606000436572).

Participants had to be over 60 years old and sedentary (no progressive resistance training (PRT); structured exercise $\leq 1 /$ week; less than $150 \mathrm{~min} /$ week low or moderate-intensity walking or other aerobic exercise). Participants could be treated with diet alone, oral medications or insulin or combination at the time of enrolment. Participants had to have stable chronic diseases, and be willing to commit to a 12-month exercise training program, 3 times per week.

Biopsies were performed at 3 time points: baseline, 6 months after training, and 12 months after training (see Figure 1). Participants were required to stop any medications that might increase the risk of bleeding subsequent to the biopsy from 7 days before the biopsy until 2 days after the biopsy. This medication list is shown in Appendix 1. Biopsies were not performed on participants who could not safely stop anticoagulants due to atrial fibrillation, mechanical heart valve, recent stent procedures or recent thrombosis/embolism. Participants took all other medications as usual on the biopsy day. Participants were allowed to refuse the biopsy and still participate in the trial.

\subsection{Skeletal Muscle and Subcutaneous Adipose Tissue Biopsy}

Percutaneous needle biopsies of the non-dominant vastus lateralis (knee extensor) muscle and subcutaneous adipose tissue were obtained under local anaesthetic (1\% Xylocaine $\mathrm{HCl}$, AstraZeneca, North Ryde, NSW, Australia) using a modification of the method described by Evans [16].

\subsubsection{Sampling Procedure}

All equipment for both sampling and specimen preparation was arranged before commencing the procedure as shown in Appendix 2. The participant was asked to rest on a bed in a reclined position with a pillow under his/her head and one pillow supporting the lateral side of the foot on the biopsy leg with the non-dominant thigh exposed. The biopsy site was swabbed with betadine solution (MCP Operations, Virginia, QLD, Australia), after ascertaining that participant was not allergic to iodine/fish/shellfish (in which case alcohol was substituted). The participant's non-dominant thigh was injected with local anaesthetic $(1 \%$ Xylocaine $\mathrm{HCl})$ into the dermis and superficial subcutaneous adipose tissue using tuberculin (dermis) and $5 \mathrm{ml}$ syringes and $21 \mathrm{G}$ needle attached by the physician. The biopsy site was left to become anaesthetized for several minutes, during 


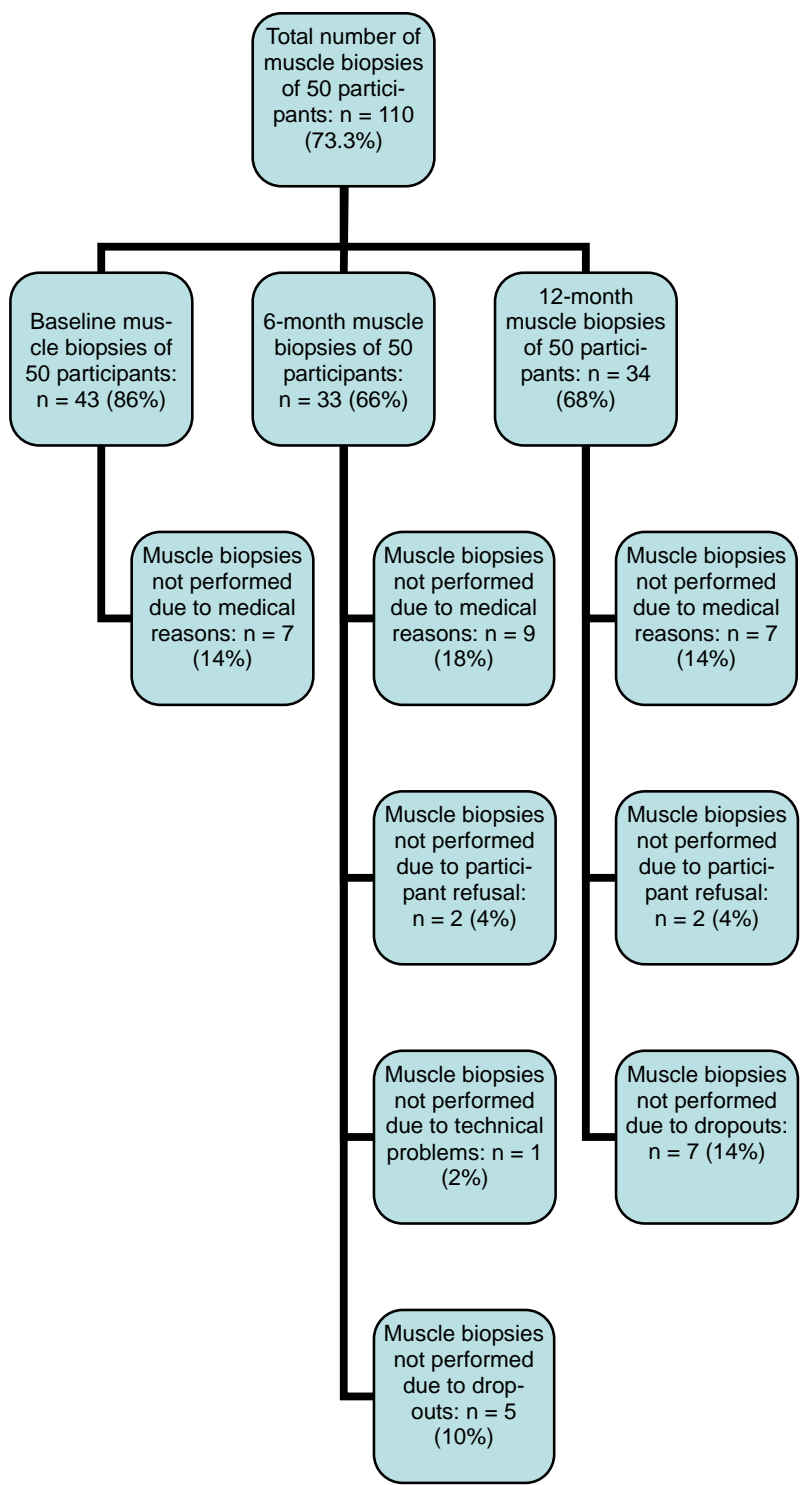

Figure 1. Flow chart of biopsies at 3 time points in 50 participants.

which time the participant was instructed to try to completely relax the muscles of the lower limb. A longitudinal incision of about $1 \mathrm{~cm}$ was made in the skin of the thigh by the physician using a scalpel. The incision was deepened so that the muscle fascia was cut if possible. The first biopsy needle was then inserted deeply into the vastus lateralis muscle by the physician. Attached to the needle were silicon tubing, a 3-way stopcock and a 50 $\mathrm{ml}$ syringe. The pipette tip connecting the silicon tubing to the needle was cut at an angle (approximately $30 \mathrm{de}-$ grees at the top) that maximized the cross-sectional area of the opening to the needle (see Figure 2). The $50 \mathrm{ml}$ syringe and stopcock were held by Assistant 1. Sampling of muscle occurred when the physician opened the window of the biopsy needle and signaled Assistant 1 to

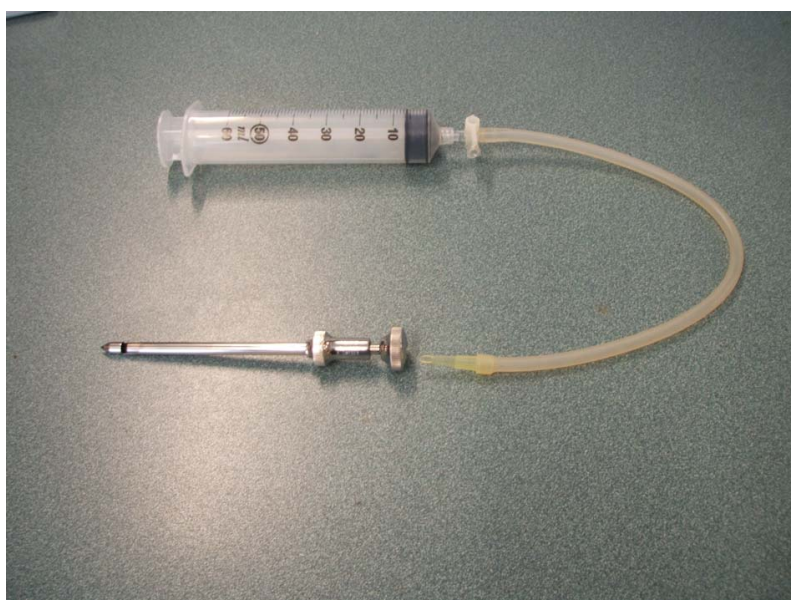

(a)

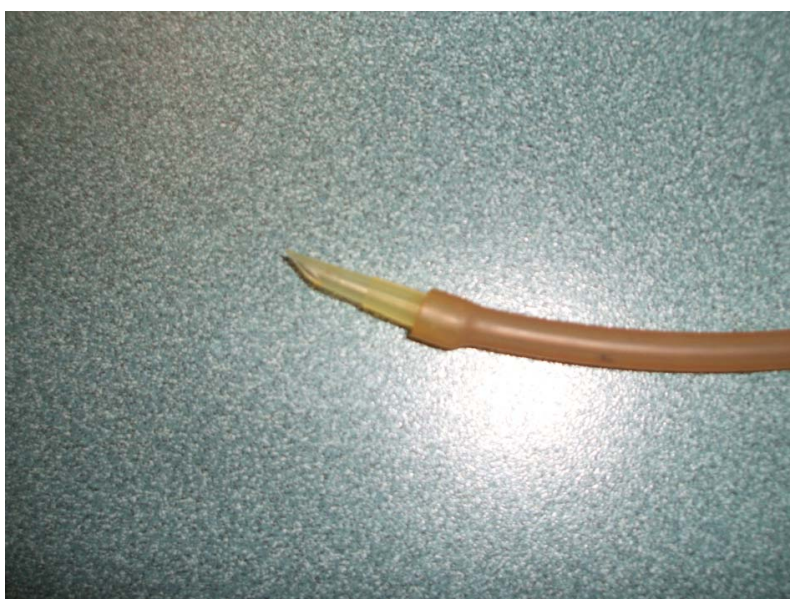

(b)

Figure 2. (a) Picture of biopsy needle, pipette tip, silicon tubing, 3-way stopcock and syringe; (b) Picture of the cut pipette tip, angled so as to maximize suction surface area and biopsy yield.

quickly and vigorously apply suction via the $50 \mathrm{ml}$ syringe. The needle window was then closed and suction was ceased. The 3-way stopcock was re-positioned by the Assistant 1 to allow air to be expelled from the $50 \mathrm{ml}$ syringe, and then the needle was repositioned slightly by quarter turns for second, third and fourth passes to be made on the thigh muscle, and then removed. After that, the second biopsy needle was inserted superficially under the skin through the same incision, and the similar procedures were used to obtain subcutaneous adipose tissue. Once sampling was completed, the muscle and adipose specimens were examined to determine whether an appropriate quantity of muscle and adipose tissue had been obtained. If the quantity of muscle or adipose tissue was insufficient, the sampling procedure was repeated with a third needle through the same incision. Pressure was applied to the incision by Assistant 2 as soon as the needle was removed for several minutes with sterile 
gauzes. The incision was then closed with Steri-strips (3M, St. Paul, MN, USA), covered with a protective pad (Cutifilm, Smith\&Nephew, Mount Waverley, VIC, Australia) and an elastic pressure (PEG) bandage (Beckton Dickinson, North Ryde, NSW, Australia) applied over a folded gauze pad positioned directly over the wound. The participant remained supine until they felt comfortable and the dressings were secure and the physician cleared them to stand. A note was made if any symptoms or complications of the procedure. The participant was advised regarding post-biopsy care and given a written copy of the care instructions (Appendix 3). The participant was called the day following the biopsy to assess any related symptoms, and generally seen within 1-3 days for the initiation or resumption of exercise training.

\subsubsection{Specimen Preparation and Storage}

Prior to commencement of the sampling procedure, isopentane was poured into a small container and placed in the liquid nitrogen canister until white balls began to form in isopentane (around $2-5$ minutes). The isopentane was not allowed to freeze.

Muscle and adipose samples were placed on the Petri dish, and cleaned with saline to remove visible blood. The muscle sample was divided into 5 samples if possible, and weighed. The samples were placed on foil, and muscle fibers aligned longitudinally, and then placed in isopentane and left for 1 minute. Then the samples were placed in the cryovial tubes, and were frozen immediately in liquid nitrogen. The adipose sample was divided into 4 samples if possible, and weighed. Then the samples were placed in the cryovial tubes, and were frozen immediately in liquid nitrogen.

After the biopsy was finished, the samples were immediately transferred from liquid nitrogen to a $-80^{\circ} \mathrm{C}$ freezer until the time of analyses.

\section{RESULTS}

Results are reported from the first 50 participants who finished the study as of Apr 2009. The average age of these 50 participants was $67.8 \pm 6.2$ years (range 59 to 83 years), $46 \%$ women. In total 110 biopsies (73.3\%) of those possible were performed on these 50 participants at 3 time points (43 at baseline, 33 at 6-month, and 34 at 12-month). The main reasons for biopsies not being performed were anticoagulation (15.3\%; participants who couldn't or forgot to stop anticoagulants), participant refusal $(2.7 \%)$, technical problems $(0.6 \%)$, and dropouts (8\%). The details are shown in Figure 1.

There were 110 biopsies, muscle samples obtained in $102(92.7 \%)$ (42 at baseline, 30 at 6-month, and 30 at 12-month), adipose samples obtained in 72 (65.5\%) (25 at baseline, 22 at 6-month, and 25 at 12-month), both tissue types obtained in 65 (59.1\%) (24 at baseline, 19 at 6-month, and 22 at 12 -month) biopsies.

The mean and standard deviation of the weight of the muscle samples was $131 \pm 92 \mathrm{mg}$ (range 3 to $424 \mathrm{mg}$ ), and the median weight of the adipose samples was 51 $\mathrm{mg}$ (range 3 to $412 \mathrm{mg}$ ). No adverse effects of biopsies were reported other than mild transient pain and light-headedness. No infections, permanent loss of sensation or other long term sequelae were observed in any participant.

High Molecular Weight (HMW) Adiponectin, Adiponectin, Tumor Necrosis Factor- $\alpha$ (TNF- $\alpha$ ), and Interleukin 6 (IL-6) were measured in homogenates of adipose tissues samples obtained in biopsies. All adipose tissue samples obtained were sufficient to analyze all four proteins, and residual sample remained for most participants.

\section{DISCUSSION}

This is one of the first reports of both adipose and muscle tissue obtained in the same percutaneous biopsy, and the first to report in detail sampling technique, yield and safety. To our knowledge, there has been only one other publication describing this method to obtain both muscle and adipose tissues from humans with and without type 2 diabetes [15], but the authors did not describe this method in detail.

It was reported by Evans and his colleagues [16] that adding suction to the percutaneous needle biopsy method (as used in this study), increased the size of skeletal muscle specimens compared with the original percutaneous muscle biopsy technique described by Bergstrom in 1962 [17]. The average weight per biopsy of the muscle samples obtained in our study (first 50 participants) was $130.6 \mathrm{mg}$, which was much higher than the average weight reported by Evans $(78.5 \mathrm{mg})$. This may be due to the increased cross-sectional area of the opening of the pipette tip we used to connect the tubing to the needle (see Figure 2), as our study physician (MFS) who learned the biopsy technique from Dr. Evans, did not cut the pipette tips at such an angle in his laboratory.

The most common reasons for biopsies not being performed were anticoagulation (16\%), and dropouts (7.3\%). Only $2.7 \%$ (4 out of 150 biopsies) of biopsies were not done due to participant refusals. This rate was lower than another similar study of resistance training in older diabetics (10 out of 62 patients or $16.1 \%$ refusal rate) reported by Castaneda [18].

\section{Limitations}

One limitation of this technique is the inability to precisely identify the site of the adipose tissue obtained, owing to the blind percutaneous biopsy procedure. This 
is important, as Goodpasteur has reported that the peripheral subcutaneous adipose tissue depot bears a different relationship to insulin resistance than the peripheral subfascial adipose tissue depot, using computerized tomography scanning (CT) to characterize the size of these depots [19]. Although we attempted to remain in the subcutaneous (i.e., above the fascia) space when inserting the second needle, in a few cases adipose tissue was obtained during the first attempt at muscle sampling, or was mixed with muscle tissue in the same needle, and could have represented subfascial/inter-muscular fat or a mix of subcutaneous and subfascial fat.

Another consideration is whether lidocaine infiltation into the subcutaneous adipocytes could have an influence on measurements obtained. As it is not possible to do a percutaneous biopsy without local anaesthesia in humans, this remains to be investigated using comparative samples from humans obtained under general anaesthesia or during liposuction, or using animal models.

\section{CONCLUSIONS}

We have shown for the first time that is possible to safely obtain sufficient quantities of both muscle and subcutaneous adipose tissue in a single percutaneous biopsy procedure over the vastus lateralis in older adults. No significant adverse events (syncope, prolonged pain, permanent loss of cutaneous sensation, bleeding, infection, hematoma, etc.) occurred despite the advanced age and presence of type 2 diabetes and multiple co-morbidities in our participants, and the refusal rate was minimal. The volume of muscle tissue obtained was not compromised by subsequent sampling of adipose tissue, and all samples were adequate for multiple analytical procedures.

Our understanding of the possibly protective role of femoral-gluteal subcutaneous fat with regards to metabolic health has been hampered to date by limited information on the morphology, biochemistry and metabolism of this fat depot, its genetic and environmental determinants, and its adaptation to lifestyle and pharmacological interventions. The application of the technique described herein within relevant clinical trials and laboratory studies will hopefully substantially advance knowledge in this field.

\section{ACKNOWLEDGEMENTS}

This study was funded by the Australian National Health and Medical Research Council, project grant 512381, Diabetes Australia, Australian Diabetes Society, and supported by the University of Sydney. Yi Wang was supported by the University of Sydney International Postgraduate Research Scholarship. We would also like to thank Dr. Patricia Ruell for the technical support, Dr. Steven Blair for the review of the article, Dr. Nasim Foroughi, Haifa Abas and Dr. Michael Baker for assistance with assessments, Jacinda Meiklejohn and Dr. Nathan de Vos for their assistance with recruitment and screening, and the participants for their generosity of time and spirit.

\section{REFERENCES}

[1] Lowell, B.B. and Shulman, G.I. (2005) Mitochondrial dysfunction and type 2 diabetes. Science, 307, 384-387. doi: $10.1126 /$ science. 1104343

[2] Meigs, J.B. (2002) Epidemiology of the metabolic syndrome. American Journal of Managed Care, 8, S283S292.

[3] Raz, I., Eldor, R., Cernea, S., Shafrir, E. (2005) Diabetes: Insulin resistance and derangements in lipid metabolism. Cure through intervention in fat transport and storage. Diabetes/Metabolism Research and Reviews, 21, 3-14. doi:10.1002/dmrr.493

[4] Rassman, J. and Gupta, S. (2005) The metabolic syndrome: Modify root causes, treat risk factors. JAAPA, 18, 30-36.

[5] Simpson, K.A. and Singh, M.A. (2008) Effects of exercise on adiponectin: A systematic review. Obesity, 16, 241-256. hdoi:10.1038/oby.2007.53

[6] Hamdy, O., Porramatikul, S. and Al-Ozairi, E. (2006) Metabolic obesity: The paradox between visceral and subcutaneous fat. Current Diabetes Reviews, 2, 367-373.

[7] Ishikawa, K., Takahashi, K., Bujo H., et al. (2004) Regulating subcutaneous fat accumulation alters insulin sensitivity. Diabetes, 53, A408.

[8] Ishikawa, K., Takahashi, K., Bujo, H., Hashimoto, N., Yagui, K. and Saito, Y. (2006) Subcutaneous fat modulates insulin sensitivity in mice by regulating TNF-alpha expression in visceral fat. Hormone Metabolism Researches, 38, 631-638. hdoi:10.1055/s-2006-954580

[9] Giugliano, G., Nicoletti, G., Grella, E., et al. (2004) Effect of liposuction on insulin resistance and vascular inflammatory markers in obese women. British Journal of Plastic Surgery, 57, 190-194. hdoi:10.1016/j.bjps.2003.12.010

[10] González-Ortiz, M., Robles-Cervantes, J.A., CárdenasCamarena, L., Bustos-Saldaña, R. and Martínez-Abundis, E. (2002) The effects of surgically removing subcutaneous fat on the metabolic profile and insulin sensitivity in obese women after large-volume liposuction treatment. Hormone Metabolism Researches, 34, 446-449. doi:10.1055/s-2002-33603

[11] Ybarra, J., Blanco-Vaca F., Fernández, S., et al. (2008) The effects if liposuction removal of subcutaneous abdominal fat on lipid metabolism are independent of insulin sensitivity in normal-overweight individuals. Obesity Surgery, 18, 408-414. doi:10.1007/s11695-007-9261-5

[12] Robles-Cervantes, J.A., Martínez-Abundis, E., GonzálezOrtiz, M., Cárdenas-Camarena, L., Hernández-Salazar, E. and Olvera-Ozuna, R. (2007) Behavior of insulin sensitivity and its relation to leptin and tumor necrosis factor-alpha in obese women undergoing liposuction: 6-month follow-up. Obesity Surgery, 17, 1242-1247. doi:10.1007/s11695-007-9213-0

[13] Kay, S.J. and Fiatarone Singh, M.A. (2006) The influence of physical activity in abdominal fat: A systematic review 
of the literature. Obesity Reviews, 7, 183-200. doi:10.1111/j.1467-789X.2006.00250.x

[14] Wang, Y., Simar, D. and Fiatarone Singh, M.A. (2009) Adaptations to exercise training within skeletal muscle in adults with type 2 diabetes or impaired glucose tolerance: A systematic review. Diabetes/Metabolism Research and Reviews, 25, 13-40. doi:10.1002/dmrr.928

[15] Plomgaard, P., Nielsen, A.R., Fischer, C.P, et al. (2007) Associations between insulin resistance and TNF- $\alpha$ in plasma, skeletal muscle and adipose tissue in humans with and without type 2 diabetes. Diabetologia, 50, 2562-2571. doi:10.1007/s00125-007-0834-6

[16] Evans, W.J., Phinney, S.D. and Young, V.R. (1982) Suction applied to a muscle biopsy maximizes sample size.
Medicine \& Science in Sports \& Exercise, 14, 101-102.

[17] Bergstrom, J. (1962) Muscle electrolytes in man. Scandinavian Journal of Clinical \& Laboratory Investigation, 14, 511-513.

[18] Castaneda, C., Layne, J.E., Munoz-Orians, L., et al. (2002) A randomized controlled trial of resistance exercise training to improve glycemic control in older adults with type 2 diabetes. Diabetes Care, 25, 2335-2341. doi:10.2337/diacare.25.12.2335

[19] Goodpaster, B.H., Thaete, F.L. and Kelley, D.E. (2000) Thigh adipose tissue distribution is associated with insulin resistance in obesity and in type 2 diabetes. The American Journal of Clinical Nutrition, 71, 885-892. 


\section{Appendix 1.}

\section{MEDICATIONS TO AVOID 1 WEEK BEFORE THE BIOPSY}

Do not take the medications on this list as of $\mathrm{dd} / \mathrm{mm} / \mathrm{yy}$.

Please continue taking all other medications as usual.

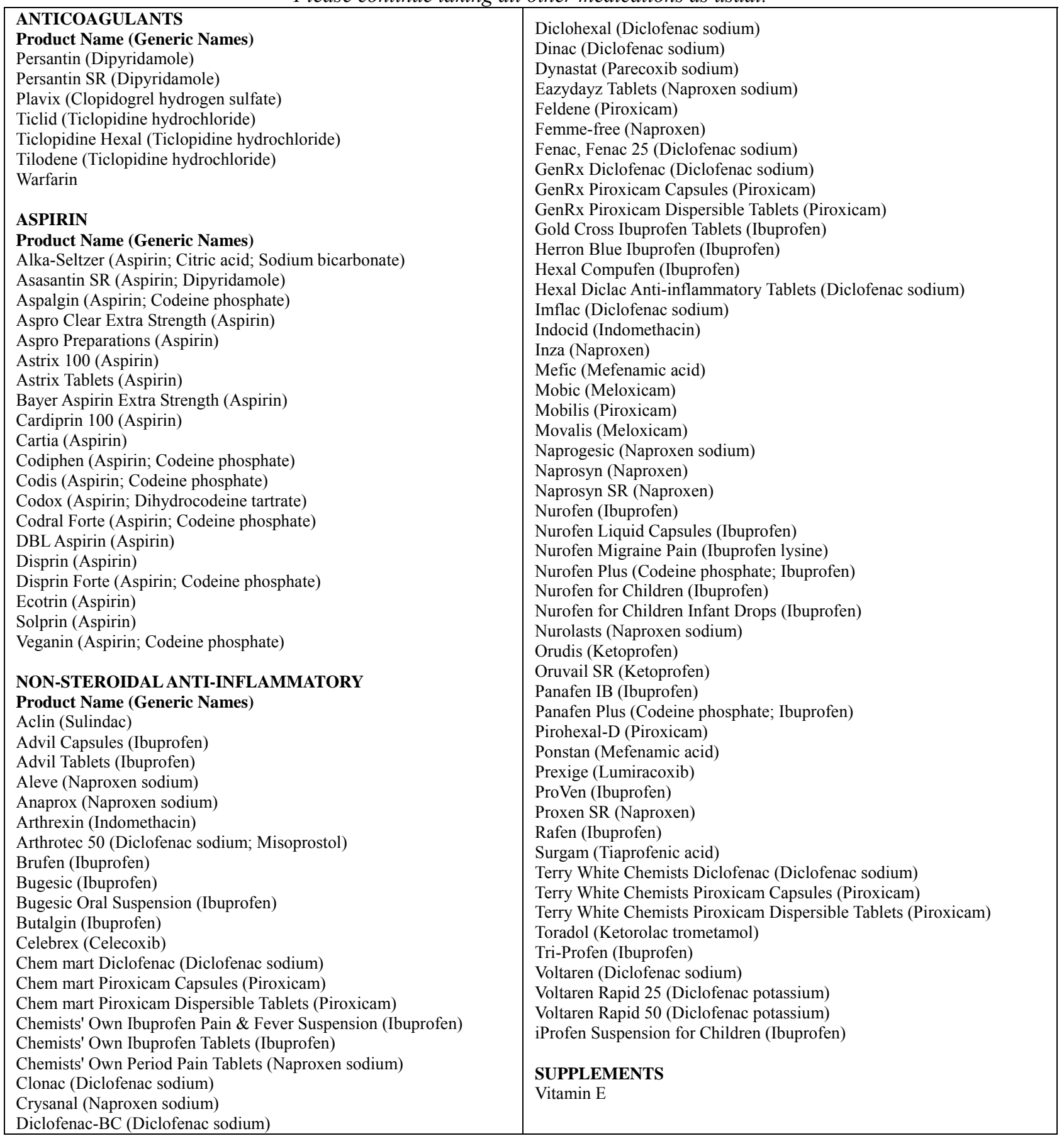


Appendix 2. Equipment required for 1 biopsy.

\begin{tabular}{|c|c|c|}
\hline & Equipment & Number \\
\hline \multirow{10}{*}{$\begin{array}{l}\text { Equipment for Physi- } \\
\text { cian }\end{array}$} & $\begin{array}{l}\text { Muscle biopsy needle kits (containing scalpel blade (size } \\
\text { 11) and handle) }\end{array}$ & 3 \\
\hline & Gloves (sterile) & 1 pair \\
\hline & Dressing pack & \\
\hline & Betadine & $10 \mathrm{ml}$ \\
\hline & PEG bandage & 1 \\
\hline & $21 \mathrm{G}$ needles & 2 \\
\hline & $5 \mathrm{ml}$ syringes & 2 \\
\hline & 50 unit insulin syringes & 2 \\
\hline & Steri-strip & 1 \\
\hline & Cutifilm & 1 \\
\hline \multirow{17}{*}{$\begin{array}{l}\text { Equipment for Assis- } \\
\text { tant } 1\end{array}$} & Incontinence pad & 1 \\
\hline & Gloves (non-sterile) & 1 pair \\
\hline & Xylocaine $1 \%$ for injection & $10 \mathrm{ml}$ \\
\hline & Scalpel blade (size 11) and handle & 1 \\
\hline & $50 \mathrm{ml}$ syringes & 3 \\
\hline & 3 way stop-cocks & 3 \\
\hline & Cryovial containers & $\begin{array}{l}\text { As } \\
\text { needed }\end{array}$ \\
\hline & Liquid nitrogen container with liquid nitrogen & \\
\hline & Isopentane & $30 \mathrm{ml}$ \\
\hline & Container for isopentane (stainless steel bowl) & \\
\hline & $\begin{array}{l}\text { Salne }(0.9 \% \text { Nacl }) \\
\text { Petri dishes }\end{array}$ & $\begin{array}{l}10 \mathrm{mI} \\
2\end{array}$ \\
\hline & Forceps & 1 \\
\hline & Aluminium foils $(1.5 \mathrm{~cm} \times 1.5 \mathrm{~cm})$ & $\begin{array}{l}\text { As } \\
\text { needed }\end{array}$ \\
\hline & Xylene free pen & 1 \\
\hline & $\begin{array}{l}\text { Container with water for immersion of muscle biopsy nee- } \\
\text { dle after procedure }\end{array}$ & 1 \\
\hline & Incontinence pad & 1 \\
\hline & Non-sterile gauzes & 10 \\
\hline \multirow{3}{*}{$\begin{array}{l}\text { Equipment for Assis- } \\
\text { tant } 2\end{array}$} & Sterile gauzes & 10 \\
\hline & Gloves (sterile) & 1 pair \\
\hline & Incontinence pad & \\
\hline \multirow{2}{*}{$\begin{array}{l}\text { Equipment for } \\
\text { ticipant } \\
\text { Other }\end{array}$} & Incontinence pad & 1 \\
\hline & Contaminated waste bin & 1 \\
\hline
\end{tabular}




\section{Appendix 3.}

Today you have had a biopsy in your thigh. It is important to observe and care for the biopsy site well to prevent any problems from occurring. Please follow these instructions carefully:

1) Keep the pressure bandage in place over the biopsy site for 24 hours. The bandage may be adjusted if it is too tight or too loose. It should remain firm but should not be cutting off the circulation and causing swelling or numbness in the lower leg or foot.

2) 24 hours after the biopsy, remove the pressure bandage and gauze pad. Wash off the yellow betadine solution if your skin is itchy, but be careful not to wet the steri-strip or biopsy incision itself.

3) Non-steroidal anti-inflammatory medicines, aspirin and anticoagulants which were on hold prior to the biopsy may be resumed after 24 hours if there is no indication of bleeding at the site. It is normal to see a little dried blood under the steri-strip and note that the skin edges do not appear completely healed at this stage. Minor soreness may also be present if the area is rubbed or touched.

4) Inspect the incision site for bleeding, swelling, inflammation, significant redness or heat in the surrounding tissues, masses (or lumps) under the skin, opening of the skin edges, oozing of fluid from the incision or significant pain or numbness near the site. If any of these signs are present, call the study physician at any time to discuss what should be done.

5) Leave the steri-strip in place until it falls off by itself. Showering or bathing of the incision site can be started 48 hours after the biopsy unless one of the problems noted in 4 above has occurred. In this case, contact the study physician for instructions prior to resumption of bathing.

6) Normal levels of physical activity may be resumed immediately after the biopsy. 